\title{
Arts, Sciences, Religions et le surdimensionnement du
} cerveau humain 2

\author{
Arts, Sciences, Religions and the over-sized human brain 2
}

\author{
Jean-Pierre Gasc ${ }^{1}$ \\ ${ }^{1}$ Museum National d'Histoire Naturelle, Paris, jean-pierre.gasc@mnhn.fr
}

\begin{abstract}
RÉSUMÉ. Parmi les religions institutionnelles, les formules monothéistes se sont imposées dans le Proche-Orient en association avec l'histoire de sociétés hiérarchisées et leurs modalités d'exercice du pouvoir. Cette relation a fait des religions des agents responsables des plus grands massacres de l'histoire. L'exercice de la raison, du doute et de la critique a conduit au développement de la philosophie et de la science, souvent en opposition avec les religions. Cependant, d'une part les causes mentales de la tentation du recours à la religion résident en chaque être humain et se trouvent renforcées par des conditions sociales, d'autre part la soif de pouvoir et l'instrumentation du fait religieux dressent des obstacles à l'épanouissement de l'humanité. Les solutions résident dans la conjonction d'une diffusion massive des connaissances et un partage volontariste des ressources.

ABSTRACT. Among the institutional religions, the monotheistic formulas were imposed in the Middle East in association with the history of hierarchical societies and their modalities of power. This relationship has made religions agents responsible for the greatest massacres in history. The exercise of reason, doubt and criticism has led to the development of philosophy and science, often in opposition to religions. However, on the one hand, the mental causes of the temptation to resort to religion reside in each human being and are reinforced by social conditions; on the other hand the thirst for power and the instrumentation of religious fact create obstacles to the flourishing of humanity. The solutions lie in the conjunction of a massive diffusion of knowledge and a proactive sharing of resources.

MOTS-CLÉS. Abraham, prophètes, messianisme, superstition, Lumières.

KEYWORDS. Abraham, prophets, messianic, superstition, Enlightment.
\end{abstract}

\section{Les monothéismes et leur dualisme implicite}

Les religions monothéistes constituent un cas particulier1, en particulier celles qui se développent successivement au Proche-Orient à partir du même schéma d'origine, symbolisé par Abraham, et dont les mythes fondamentaux consignés dans la Bible au sens large continuent à être pratiquées.

Historiquement, il semble que le monothéisme soit d'abord passé par une phase où une des divinités reconnues par la société ait été mise au premier plan. Ensuite, elle aurait été reconnue comme exclusive et le panthéon définitivement rejeté. Pour tenter d'expliquer ce phénomène, il convient encore de distinguer le plan individuel, personnel, et celui du groupe ou d'une fraction qui a la charge des rites. D'un point de vue individuel, les tourments nés de l'hyperactivité cérébrale, les interrogations sur la mort et la peur du monde imaginaire qui est bien ressentie individuellement, le poids de l'inconscient, ne sont jamais totalement satisfaits par le recours à l'animisme et ses superstitions, ni par les mythes et sacrifices à de multiples divinités qui appartiennent aux traditions de la communauté. Cette insatisfaction nourrit la permanence du recours de la religion et de ses pratiques souvent contraignantes et complexes, ce qui constitue une véritable addiction se manifestant par une superstition aigue qui pèse sur la vie quotidienne. Précisément, puisqu'il y a servitude n'est-il pas avantageux, simple et peutêtre plus efficace de s'en remettre à une seule entité sur laquelle se concentre tous les rituels ? Et sur le plan du ressenti introspectif le sujet se voit alors en contact avec une seule force. Le passage vers une abstraction, un "non-nommé », est alors possible dans la fraction initiée du groupe, ce qui fait du monothéisme une religion pour les élites. Cependant, une difficulté récurrente s'est soulevée sur la voie conduisant au monothéisme, et a produit parfois une vive opposition des masses. En effet, la diversité des situations inattendues, inexplicables, insolites, conduisant à des drames, à des morts, maladies et catastrophes ne paraissent pas pouvoir être justifiées, ni par des forces naturelles impersonnelles ni par un seul être surnaturel. Face à une typologie des maux de l'existence, il est plus 
facile, dans le cadre d'une mentalité où les relations de causalité ne sont pas perçues, de placer des « génies » en regard de ces « accidents de la vie », une série de puissances spécialisées en vertu d'une continuité entre l'homme et l'ensemble de la nature. Alfred Métraux (1958) rapporte comment le Dieu unique du christianisme cohabite en Haïti avec les esprits craints et vénérés par le culte du vaudou, que rien n'a pu déraciner. Ces esprits et le culte associé sont ressentis comme tellement nécessaires que leur rejet s'accompagne généralement de troubles graves de la santé mentale. Il semble ainsi que les êtres surnaturels qui sont présumés avoir un impact quotidien sont considérés comme des agents stratégiques, contrairement aux «grands créateurs » trop éloignés du quotidien. Ils alimentent les superstitions que combattent alors les doctrinaires, (Boyer,2001). La lecture de la Bible nous apprend sous forme mythifiée combien il avait été long et difficile d'instaurer le monothéisme strict dans les tribus de l'ancienne Palestine. C'est semble-t-il la cruelle épreuve de l'exil de tout un peuple à Babylone et en Egypte qui a été déterminante dans le rejet définitif du polythéisme. L'argument décisif fut un retournement du jugement porté sur la défaite d'Israël. Au lieu de faire naître un sentiment de rejet face à une divinité (Jahvé ou YHVH) qui paraissait avoir abandonné ses créatures, les chefs religieux ont mis en avant la culpabilité du peuple, et ont présenté ces revers comme un châtiment en raison de la rupture de l'Alliance, preuve de la sollicitude du créateur et de la place prééminente des Juifs, soumis ainsi à une épreuve destinée à resserrer les liens privilégiés que ce peuple entretient avec le créateur. Ce fut en même temps l'occasion de créer un soutien sans faille au pouvoir des rois.

L'histoire semble montrer que le monothéisme n'a pu être imposé durablement sans l'adhésion soit du collège des prêtres, soit des peuples subjugués par des prophètes. Ainsi, en Egypte, au $14^{\mathrm{e}}$ siècle avant notre ère, Aménophis IV imposa un culte monothéiste, celui du soleil (Aton) et pris le nom d'Akhenaton. Mais ce brusque abandon d'une tradition déjà millénaire ne résista pas après la mort du pharaon à la pression de toute une hiérarchie de prêtres et de dignitaires qui avaient perdu tout pouvoir et richesse. Le rôle des prophètes, personnes s'imposant aux masses par la parole, paraît essentiel à la survie des monothéismes, fragiles devant la pression des rituels anciens et de la personnalisation des forces naturelles, des accidents de la vie et des fantasmes de l'inconscient qui poussent vers l'animisme. C'est à plusieurs reprises que la religion des tribus d'Israël est sauvée par le discours culpabilisant de prêcheurs qui jouent en outre du ressort divinatoire et d'un réel pouvoir de suggestion pour susciter un élan messianique tout spécialement en période de crise.

Une autre difficulté mine le monothéisme strict. Ce Dieu unique est d'abord bon, bienveillant et miséricordieux, il doit séduire et rassurer. Mais cette image est bien vite difficile à défendre sans un aveuglement complet, une servitude volontaire. Le Mazdéisme venu sans doute de l'Inde vers l'Iran ancien et prolongé par Zoroastre montre l'exemple d'un monothéisme oscillant pendant des siècles entre son rejet initial des rituels sacrificatoires et la prise de drogues hallucinogènes et une conception dualiste dans laquelle un dieu bon fait face, comme l'image modifiée dans un miroir truqué, à un dieu méchant. En effet, au niveau individuel et de la famille nucléaire la vie offre trop de drames qui paraissent injustes et l'auto-culpabilisation prônée par la doctrine ne suffit pas à effacer les interrogations sur l'efficacité d'un dieu réputé bon. Ceci conduit à la supposition soit de l'abandon des créatures par le créateur, soit à l'existence d'un dieu malin, dans tous les sens du terme. Les courants d'un dualisme plus ou moins accentué (manichéisme, gnosticisme, catharisme, sont les plus célèbres) ont régulièrement menacé les monothéismes institutionnalisés. Contrairement à leur rationalité apparente - expliquer l'existence des effets néfastes de la vie par une cause unique, soit suprême et égal au dieu bon, soit rebelle («ange déchu») mais laissée libre d'agir - les mouvements dualistes ouvraient sans le vouloir les portes aux anciennes superstitions, aux génies et autres diablotins, plus proches de l'expérience individuelles des divagations cérébrales. D'où l'ambiguïté des sculptures ornant les chapiteaux et les modillons des églises romanes où dominent les représentations de créatures imaginaires et inquiétantes par leur monstruosité et leurs attitudes souvent agressives.

Mahomet (Muhammad), originaire de La Mecque en Arabie, s'inscrit dans la lignée des prophètes de la religion d'Abraham. Il reproche aux hommes de s'être une fois de plus écartés de la vraie foi, six siècles après l'intervention de son prédécesseur, Jésus. Il a voulu refonder le monothéisme du livre 
saint, la Bible, mais dans le contexte particulier de l'Arabie, où prospèrent au $7^{\mathrm{e}}$ siècle des tribus nomades qui profitent de la circulation des marchandises par les voies du désert entre l'Afrique et l'Asie. Contrairement à la plupart des autres prophètes, Mahomet apparaît sous les traits d'un fin politique. Il ne va pas suivre l'exemple de l'échec d'Israël face à l'empire romain, ni celui de la réussite d'un christianisme récupéré en devenant religion de l'empire en 380 par l'édit de Thessalonique prononcé par Théodose 1er. Cette refondation rend nécessaire une diffusion massive de sa pensée. Au cours d'un exil de 10 années à Médine, il dicte l'essentiel du Coran, recueil des révélations divines (par l'intermédiaire de l'ange Gabriel, car dieu ou Allah ne peut être personnifié) assorties de réflexions et conseils sur la vie individuelle. Loin de faire de ce livre un conservatoire plus ou moins réservé à une élite de prêtres, il en fait le guide obligatoire de chaque croyant, écrit dans la langue locale, l'arabe. Son action se déroule dans un milieu tribal, sans état, c'est-à-dire dans le jeu d'intrigues et d'échanges de services et de concurrence d'influences entre marchands, ce qu'il est à l'origine. Il sera menacé en tant que prophète non pas par un pouvoir centralisé appuyé sur un corps de prêtres ou de guerriers, mais par des conservateurs jaloux qui craignent tout changement comme pernicieux pour leur commerce. Le charisme de Mohamed lui permet de réunir suffisamment d'adeptes résolus pour employer la tactique propre au désert : la razzia contre les caravanes de ses détracteurs. Puis il prend d'assaut La Mecque, centre important du commerce. Du même coup, il annexe un culte préhistorique vénérant une pierre noire tombée du ciel, météorite appelée Kaaba. Commence alors une extension fulgurante de l'Islam (qui signifie « sujétion ») par la force des armes et de la parole, un culte qui n'exige qu'un rituel individuel quotidien et qui est strictement monothéiste, abstrait même, plus encore que le judaïsme, et qui rejette la personnification. Mohamed lui-même n'est que le prophète d'Allah, un messager qui meurt et dont la vénération ne viendra que plus tard. La désignation d'un successeur, ou calife, ne posera de problèmes qu'à la seconde génération, car c'est à la fois un chef religieux et un chef de guerre, et ce sera alors le début d'une guerre sans merci entre chiites qui fondent leur légitimité sur la désignation d'Ali par Mahomet lui-même de son vivant, et les sunnites qui prennent ensuite le pouvoir politique par la violence. Cependant, malgré la multiplication des sectes au fil des siècles l'Islam ne cessera d'étendre son emprise politique sur des peuples non-arabes. Le pouvoir y apparaît comme une délégation de celui qu'exerça le prophète, ce qui ouvre évidemment la voie à toutes les manipulations. Il n'y a pas de hiérarchie institutionnelle, du moins à l'origine, et comme chacun peut s'autoproclamer prédicateur ou dignitaire, il fut rapidement nécessaire d'instaurer un système d'enseignement particulier pour préserver la doctrine, qui s'exprime en arabe. La cohésion repose d'une part sur le livre, sa langue et le rituel, d'autre part sur une administration rigoureuse des choses et des hommes qui rappelle le cas chinois, issue aussi d'une pratique marchande. La simplicité structurelle et la quotidienneté rituelle ont certainement compté dans le pouvoir de séduction de l'Islam, en plus de la sidération des peuples conquis. Cependant, une part du dualisme biblique, l'existence de Satan un ange déchu, est conservé, mais il joue le rôle d'identifiant des ennemis, et des rebelles incroyants. Ceci révèle d'ailleurs une contradiction que le judaïsme n'avait pas résolue non plus. En rejetant les ennemis du côté du Mal, on nie le côté universel et bienveillant du créateur, on définit deux catégories de créatures et on ouvre donc la porte au fanatisme meurtrier. Comment concilier cette attitude avec le monothéisme strict et les consignes de solidarité envers les faibles et de bienveillance fraternelle envers tout humain que déclare la doctrine ? La différence sur ce point avec le judaïsme orthodoxe est que la conversion à l'islam suffit à entrer dans la communauté des croyants (ummah). Il n'y a pas de peuple élu, d'où l'expansion étonnante de l'islam.

Dès que le christianisme devint en 380 religion d'état par l'édit de Thessalonique sous l'empereur Théodose 1er, il tourna aussi le dos au monothéisme strict, et progressivement, jusqu'à la réforme grégorienne du douzième siècle, l'Eglise se confondit avec les pouvoirs politiques, jusqu'à les supplanter dans les domaines de la vie des individus. D'assemblée des fidèles, l'église chrétienne s'est transformée en institution, avec une organisation hiérarchique et une distribution territoriale, les évêchés concentrant pouvoir politique et substrat économique. Les seigneurs, représentants de la caste guerrière, perdent du pouvoir et des ressources face aux évêques et aux monastères. D'une certaine façon l'église romaine a sauvé momentanément l'Empire d'occident lorsqu'il tombe en miettes, puis par le jeu de l'acculturation des envahisseurs et l'infiltration missionnaire en vue de conversion, elle est 
devenue le soutien idéologique des souverainetés européennes. Sa position hégémonique qu'est venue renforcer l'accroissement des pouvoirs du Pape élu à partir de 1059 par les cardinaux et non plus par l'Empereur, ouvrit là encore la porte au fanatisme. Le grand schisme intervient en 1054 entre Rome, qui se veut hégémonique parce qu'héritière de l'apôtre Pierre, et Byzance où Empire et église restent étroitement associés. La religion plus que jamais s'illustra comme un prétexte dans les prises de pouvoir, et les massacres. C'est ainsi que l'aristocratie guerrière totalement apprivoisée par l'idéologie romaine aura son énergie utilisée dans les opérations des croisades, prétexte d'une libération des lieux saints ou de l'élimination des hérésies. L'une de ces croisades est particulièrement significative de cette dérive de la religion catholique qui, pour défendre son socle dogmatique, n'hésite pas à se comporter en puissance tyrannique, car la croisade contre les Albigeois eut lieu à l'instigation du pape Innocent III au début du $13^{\mathrm{e}}$ siècle sur les terres de la France en train de se consolider. En furent victimes les masses habitant l'Occitanie qui défendaient leur pratique d'une foi religieuse se voulant proche du christianisme originel, et qui rejetaient donc la réforme grégorienne et ses institutions. Celles-ci en effet avaient officialisé une superstructure faisant écran entre le croyant et la divinité, une hiérarchie ecclésiastique qui, du prêtre au pape, étendait son administration, son pouvoir économique et son emprise sur la vie des individus. Mais l'inquisition, bras religieux de la répression contre le catharisme, n'est fondée à Toulouse qu'en 1233, c'est-à-dire après l'intervention sanglante de l'aristocratie guerrière du nord dont le véritable mobile a été le pillage et la spoliation des terres du comté de Toulouse. Cette région était en effet convoitée à la fois par Henri Plantagenet et le capétien Louis VII qui y voyaient une façon d'accroître leur domaine royal respectif d'Angleterre et de France. C'est aussi l'époque, dès 1 e $12^{\mathrm{e}}$ siècle, où apparaissent des écrits violemment antisémites et anti musulmans désignant des boucs émissaires ce qui entrainent des massacres populaires alimentés par des rumeurs à l'occasion d'épidémies. On voit là au niveau de peuples, la restriction de la qualité d'humain aux membres de la communauté, au dehors il n'y a plus d'humains. Il s'agit d'un phénomène qui s'observe au niveau de tout groupement humain, dans toutes les sociétés, et qui se traduit très souvent dans le langage, par la restriction de l'usage de terme désignant les hommes aux seuls membres du groupe soumis au rituel. Et alors tout est possible, puisque les règles de la communauté ne sont plus applicables à l'extérieur. Cimenter des communautés fut en effet un des rôles historiques des religions, et l'une des conséquences a été de justifier le massacre massif des autres.

La récurrence au Moyen Age des schismes qui jusqu'à la Réforme réclament un retour aux sources évangéliques et sont qualifiés d'hérésies par l'église romaine, moyen commode d'éviter le dialogue ou la conciliation, révèle cependant la réalité d'un malaise vis-à-vis de la dérive du monothéisme institutionnel. Les réponses furent de façon constante des massacres de masse, ce qui fait dire à Elias Canetti (1966) :«il n’y a jamais eu sur terre un seul état qui s'entendit mieux à se défendre contre la masse. Comparés à l'Eglise, tous les souverains font figure de piètres amateurs ». On peut noter d'ailleurs que, comme toute secte parvenue au pouvoir, les religions issues de schisme se sont révélées aussi intolérantes et sanguinaires que les premières religions monothéistes.

\section{La défense de l'individu}

Cependant, ces soubresauts des dogmes qui ont émaillé l'histoire ne doivent pas masquer « qu'en bas », dans les masses populaires majoritairement rurales, des cultes antiques, hérités de la préhistoire, ont continué à être pratiqués, jusqu'à contaminer le monothéisme chrétien, particulièrement sensible au processus de personnification. C'est ainsi que malgré une transformation opportuniste par l'église des héros locaux en « saints » et la pose de croix sur des sites naturels dont l'antique usage rituel était ainsi «christianisé », les pratiques considérées comme «païennes » se sont poursuivies. André Varagnac, théoricien du folklorisme, a décrit ce glissement en surface du christianisme sur ce qu'il appelle une «archéocivilisation» (1948) dont il voyait les traces dans le folklore et la vie quotidienne des masses européennes rurales jusqu'au début du 20e siècle. Des pratiques typiquement païennes avaient encore cours dans les années 1970, comme j'ai pu le constater à deux reprises. Une première alors que je visitais une chapelle isolée dans la lande du pays bigouden, et que le jeune prêtre qui m'en avait ouvert la porte me confia qu'il avait été horrifié par la demande de certains habitants de voir bénir leurs 
animaux ; la seconde lorsque hébergés par le curé du village au cours d'une fouille paléontologique dans la Beauce, nôtre hôte nous confia que des rites continuaient à se dérouler dans des cavités naturelles de la région. Boyer (2001) observe aussi que les peuples s'écartent souvent du «théologiquement correct » défini par les religieux institutionnels. A partir de ce siècle la vie urbaine a emporté les fêtes rythmant les saisons et les communautés villageoises autour des sites naturels, fontaines, roches ou grottes. Cette disparition est accompagnée par la réduction de la pression institutionnelle de la religion, du moins en France. Mais cette sécularisation de la société n'a pas effacé la pression du travail à vide de notre cerveau qui, par un processus toujours identique, continue depuis les origines humaines à offrir à chaque individu cette vie parallèle qui nie les relations de causalité et concurrence l'exercice de la raison sur le monde réel. Il suffit de consulter aujourd'hui le succès des annonces de services proposés par des devins, guérisseurs et autres charlatans. L'incorporation sociale, dans les structures de la société par les mythes et les rituels, du fait religieux individuel souvent réduit à la superstition, agit comme une manière d'échapper à un destin individuel et permet d'exister en tant qu'être humain au travers du groupe social. Répétons ici que ce processus répond à la fois à un besoin individuel et à un besoin social de l'individu. C'est aussi un facteur de cohésion du groupe humain. C'est pourquoi ce processus a dû jouer un rôle non négligeable dans l'émergence des qualités propres aux premières populations humaines, dès lors qu'un langage minimal a permis un échange d'information entre individus. C'est une manière de gommer la contradiction pénible entre réel et imaginaire, un soulagement pour l'esprit : "Si le surnaturel est senti comme réel, en effet, s'il se mélange au réel dans le mythe, c'est que le mythe est participation vécue, et non-participation pensée, c'est qu'il n'est pas un produit de l'activité mentale en tant que cognitive, mais en tant que sentiment expérimenté ». (Roger Bastide, 1968). Voilà aussi les portes de l'enrôlement lent dans l'orbite des sectes par le biais d'une persuasion jouant essentiellement sur le registre affectif, sur les émotions et un sentiment d'abandon. Historiquement, la révolution industrielle et l'essor du capitalisme ont créé les conditions d'une aliénation des individus par le biais du travail et de la consommation ne laissant plus de place pour une utilisation complète des capacités cérébrales, qui sont alors dérivées vers la facilité d'un marché du rêve programmé. Même les substances psychotropes qu'utilisaient les rituels chamaniques et antiques sont détournées pour une fuite individuelle vers «les paradis artificiels » (de Felice, 1936).

Mais, comme nous le disions au début de ce propos, il existe d'autres voies d'exutoire pour ce surplus d'activité cérébrale. Dans une société où l'individu peut librement exercer sa raison et trouver les éléments de rationalité pour appréhender objectivement l'essentiel des phénomènes agissant sur le monde réel et accéder ainsi à une compréhension permettant d'organiser ses actions dans une chaîne causale, le surplus d'activité neuronale, cet ensemble d'association gratuites qui échappe à son contrôle et lui forge pendant le sommeil cette vie imaginaire dont il prend conscience au réveil, lui ouvre aussi les portes de la création artistique comme de l'intuition scientifique. Ainsi coexistent chez un même individu deux activités mobilisant des fonctionnements distincts de la machinerie cérébrale, ce qu'avait bien exposé Roger Fry dès 1909 (1951). En effet, bien que dans son essai sur l'esthétique ce critique anglais traite surtout des arts picturaux, il tente de dégager une théorie générale de l'art en se fondant sur le rôle de ce qu'il appelle « the imaginative life », déconnectée du monde réel, et source d'une recréation.

«Ainsi, l'art est une expression et un stimulus de la vie imaginaire, qui se distingue de la vie active par l'absence d'action en réponse. En fait cette action en réponse implique dans la vie réelle une responsabilité morale. En art nous n'avons pas une telle responsabilité morale - il offre une vie libérée de toutes les contraintes de notre existence réelle. » (Notre traduction). 




Jérôme Bosch, le jardin des délices, panneau central, détail. Musée du Prado.

\section{La nécessité des Lumières}

Enfin, l'esprit humain se caractérise par une autre attitude face aux interrogations que lui suggère sa conscience supérieure. «Aie le courage de te servir de ton propre entendement ! Voilà la devise des Lumières » écrit Kant à la fin du $18^{\mathrm{e}}$ siècle. L'humain est avant tout un curieux et il ne peut lui échapper que certains événements sont toujours précédés par d'autres et qu'il dispose ainsi d'une cohérence qui n'a pas besoin de faire appel au surnaturel ou à des mythes, qui sort du carcan des dogmes et échappe à l'emprise du rituel. Contrairement à l'injonction des dogmes qui se résume à un « circulez, il n'y a rien à voir », et qui exigent de se soumettre à des réponses simples et définitives, la prise de conscience des relations causales entre des éléments du monde matériel et les capacités nouvelles que l'individu peut en tirer ouvre le champ de la réflexion, celui de la constitution d'un corpus d'idées distinctes, même si celles-ci furent au cours de l'histoire, souvent partiellement incluses dans les traditions de nature religieuse. Ainsi est née une pensée où se sont trouvées mêlées pendant longtemps ce qu'on nomme aujourd'hui la philosophie et la science, pensée caractérisée par l'exercice $\mathrm{du}$ raisonnement et la production de règles ou guides dans le processus de la connaissance. De cette double vie ressentie par chacun naissent aussi les questions fondamentales et récurrentes de la philosophie : 1. La réalité a-t-elle une existence en dehors de 1'esprit humain ? 2. Si oui, comment l'appréhender et la connaître ? On comprend de suite que cette voie d'activité mentale, ou plutôt les résultats de cette activité et leur expression par le langage, est entrée en contradiction avec toute forme de religion institutionnalisée. Un très long combat, plus qu'un débat, a donc surgi sans doute depuis la nuit des temps, dont nous n'avons que les traces écrites assez récemment. C'est en effet par la pensée des philosophes grecs d'Ionie, au 6 e siècle avant notre ère, connus sous le nom de « physiciens », que nous trouvons un courant où un savoir « désacralisé » apparaît (Vernant, 2004). Physiciens, car ils mettent au premier plan la nature ( $\varphi v ́ \sigma i \varsigma)$ et tous ses phénomènes dans le cosmos dont ils recherchent l'ordre par la géométrie et non pas par l'action de héros mythiques. Thalès, Anaximandre, Anaximène, Héraclite diffusent leur pensée grâce à une langue disposant d'une écriture alphabétique, le grec, échappant au secret d'une caste de scribes. Ils n'ont eu à craindre ni tyran ni grands prêtres, car ils vivaient dans des cités dont l'organisation politique était nouvelle, où le pouvoir électif est partagé entre les hommes d'une communauté qui est fondée sur un ordre politique (celui de la cité), des règles débattues et instaurées par les hommes eux-mêmes (du moins ceux qui étaient des mâles libres) et qui ne découlent pas directement de mythes. Cette période laissa une trace indélébile dans les esprits durant des siècles, malgré sa brièveté et les régimes tyranniques et l'impérialisme romain qui lui ont succédé. Le monde européen d'aujourd'hui doit tout à ce mouvement qui fut continuellement en butte aux poursuites des religions instituées à qui en revanche nous sommes redevables de tant de massacres.

\section{Que faire aujourd'hui ?}

Il est tout à fait contre productif de vouloir comparer les dogmes religieux à nos connaissances actuelles, même imparfaites, sur l'évolution de l'univers, monde vivant compris. On pourrait soupçonner que ce ne sont pas les mêmes circuits neuroniques qui produisent d'un côté le besoin d'un recours commode aux croyances et de l'autre la pulsion aventureuse vers un démontage des chaînes de 
causalité qui ordonnent notre univers. Fort heureusement, la réflexion philosophique d'une part, et la création artistique sous ses diverses formes d'autre part, ont pu se développer en marge des religions, parfois à leur ombre, souvent en opposition. Bachelard (1938) préconise de «penser contre son cerveau ». Voilà qui rejoint notre idée que le surdimensionnement de notre cerveau conduit à une dualité qui se manifeste par le sentiment d'une double vie, vie réelle et vie imaginaire. Ainsi pour s'assurer d'un comportement conforme à la raison, et dans le cas évoqué par Bachelard, de pratiquer l'usage de l'esprit scientifique, il convient d'éviter tout lien, toute influence en provenance des produits de cette partie du cerveau qui nous échappe et qu'on peut néanmoins valoriser sur un autre registre. De manière plutôt paradoxale, cette lutte est devenue historiquement consciente et, éclairée par la connaissance, a produit dès les premiers âges un effort de «normalisation » par le jeu des croyances au surnaturel, mais aussi par la création artistique, création d'une «nature parallèle ». Le tourment des créateurs comme celui des mystiques en est la conséquence. L'erreur du positivisme est d'avoir cru à une gradation irréversible vers le triomphe de la raison universelle. En réalité, c'est à chaque génération, dans le cerveau de chaque humain que cette lutte a lieu, dans le contexte particulier des diverses sociétés dont les éléments structuraux issus de l'histoire de chaque groupe humain constituent des guides, néfastes ou bienveillants, qui renforcent les difficultés ou bien les atténuent, comme en témoignent la diversité des cultures.

L'organisation de nos neurones dans l'encéphale nous permet une compréhension encore limitée du monde matériel, par l'exercice libre d'une recherche des chaînes de causalité. C'est cette part issue de notre surdimensionnement cérébral à laquelle nous devons donner une forme exclusive d'attention face à la complexité de la réalité. Bien entendu, il faut être conscient que, malheureusement, le désarroi de très nombreux individus les poussera encore pendant longtemps vers les illusions religieuses et la simplicité de leurs promesses, et que celles-ci prises comme prétexte, tenteront de se saisir du pouvoir pour entraîner des masses vers un destin suicidaire. A la fin du $19^{\mathrm{e}}$ siècle, Kant, écrit encore : « Après avoir rendu tout d'abord stupide leur bétail domestique, et soigneusement pris garde que ces paisibles créatures ne puissent oser faire le moindre pas hors du parc où ils les ont enfermées, ils leur montrent ensuite le danger qu'il y aurait à essayer de marcher tout seul. Or le danger n'est sans doute pas si grand que cela, étant donné que quelques chutes finiraient bien par leur apprendre à marcher ; mais l'exemple d'un tel accident rend malgré tout timide et fait généralement reculer devant toute autre tentative. Il est donc difficile pour chaque individu de sortir de la minorité, qui est presque devenue pour lui nature ».

On aurait pu espérer que les pulsions irrationnelles entrent lentement en extinction, après la généralisation de l'enseignement des enfants dans la plupart des pays, et les effets matériels de l'exercice de la raison, les réalisations technologiques issus de l'avancement des connaissances sur les processus matériels qui animent le monde naturel. Mais dès son début le $21^{\mathrm{e}}$ siècle montre tout le contraire. C'était en effet sans compter avec les alliés puissants de l'obscurantisme que constituent la cupidité et la soif de pouvoir. Les exemples de Gandhi et Mandela ont été bien vite oubliés. De plus, la science, qui est totalement neutre sur le plan des valeurs, a procuré, par ses dérivés technologiques, des moyens d'agir qui sont devenus des enjeux dans les effroyables conflits du $20^{\mathrm{e}}$ siècle. Le positivisme et sa religion du progrès, l'illusion du scientisme, ont largement contribué à l'aveuglement des hommes de science. En réaction il s'est développé un rejet de la science par la population, c'est-à-dire un rejet de la rationalité, même dans les domaines où les retombées positives sont évidentes, comme dans celui de la santé. Enfin, engendrés par les stupidités géopolitiques des impérialismes et totalitarismes du $20^{\mathrm{e}}$ siècle que n'ont pu ou su éviter les révoltes, quand elles ne les ont pas instrumentalisées, des conflits entre peuples se déclenchent à présent sous prétexte de religion. Ainsi se décline la recette tant de fois expérimentée au cours de l'histoire : l'inquiétude et le malaise des individus sont aspirés par une construction socialisée offrant le soutien de mythes et rites. Une communauté est ainsi constituée, disponible pour toute manipulation visant à éliminer physiquement les «autres », jusqu'à la folie suicidaire. De plus, il ne s'agit pas à présent d'obtenir simplement l'adhésion par le rassemblement d'individus en un lieu, une localité, la conversion d'une cité. La puissance des communications à distance a bouleversé la pratique et c'est par la communication électronique que se crée une vaste 
communauté d'abord virtuelle, dans la préparation d'un rassemblement promis. Le phénomène est général, propre à tous les mouvements monothéistes et contaminent même les autres grandes religions historiques, comme l'hindouisme. Il n'est pas indifférent que dans la sphère politique tous les mouvements se réclamant d'un nationalisme intègrent aussi des courants religieux intégristes. Depuis le $19^{\mathrm{e}}$ siècle, les églises ont toujours soutenu les nationalismes, se substituant même parfois à l'idée de nation lorsque l'état souverain disparaissait comme l'illustre l'Histoire de la Pologne. De même, l'église orthodoxe russe est à présent littéralement associée au pouvoir du Kremlin. Forte de ses biens retrouvés, elle construit de somptueuses églises dont l'architecture et la décoration sont des fac-similés de l'ancienne époque, témoignage direct de sa volonté de s'opposer à tout changement et de soutenir ainsi un pouvoir autocratique, comme au temps des Tsars. Répétons que dans tous les cas la religion n'est qu'un prétexte, ne nous y trompons pas, pour s'assurer un pouvoir et en tirer un bénéfice matériel en soumettant des humains à la volonté d'autres. Le moteur de ce processus de servitude volontaire réside dans le malheur des individus ou le sentiment d'être malheureux. Sur ce plan, les armes ne sont pas égales face à l'obscurantisme et tant que les religieux sont au pouvoir, la partie est perdue. La solution est donc politique. Il est obligatoire et sans concession possible d'établir une séparation inamovible entre les institutions à caractère religieux et celles qui sont chargées d'organiser la vie des individus en un tout social. La liberté de conscience doit être garantie par le pouvoir, ce qui signifie qu'il ne peut intervenir dans l'expression artistique ni dans l'orientation des sciences. La démocratie sous sa forme représentative est très vulnérable si on ne développe pas d'une part un effort considérable dans l'information scientifique et l'éducation permanente. Il est dangereux de laisser se développer l'ignorance des principes de base de nos connaissances du monde matériel, laissant ainsi la place aux pires manipulations régressives et au négationnisme., D'autre part, une attention de chaque instant doit être portée à tout frein dans la répartition des fruits du travail, c'est-à-dire qu'il faut enfin instaurer les règles d'une juste redistribution des richesses. L'humanité n'a pas d'avenir en dehors du partage.

Or, il faut reconnaître que les décideurs politiques, même quand ils sont portés par le suffrage des votants, ne montrent aucune volonté à mettre sur pied une protection de la démocratie, par une véritable mise en application de ses principes fondamentaux. Le manquement aux principes d'éducation et de justice sociale fait le lit des démagogues qui utilisent les règles de la démocratie dans le but avoué de la démanteler et ouvre ainsi la voie aux régimes autoritaires. On sait par expérience de l'histoire que les premiers actes de ceux-ci lorsqu'ils sont parvenus au pouvoir sont de prendre le contrôle des consciences, de la science et des arts, en trouvant vite un appui dans le camp religieux quand celui-ci n'a pas participé à l'anesthésie des peuples.

\section{Références}

Bachelard G. 1938. La formation de l'esprit scientifique. Librairie philosophique J. Vrin, Paris. (réédition en 1996).

Bastide R. 1968. La mythologie.In: Ethnologie générale, Bibliothèque de la Pléiade, Paris, Gallimard.

Boyer P. 2001. Et l'homme créa les dieux. Comment expliquer la religion, Paris, Robert Laffont.

Canetti E. 1966. Masse et puissance. Paris, Gallimard.

Felice P. de. 1936. Poisons sacrés Ivresses divines : Essai sur quelques formes inférieures de la mystique. Paris, Albin Michel.

Fry R. 1961. An Essay in Aesthetics, 1909, In : Vision and Design, London, Penguin Books.

Kant E. 1784. Beautwortung der Fraze : Was ist Aufklärung ? Berlinische Monatschrift.

Krappe, A.H. 1952. La genèse des mythes. Paris Payot.

Lévi-Strauss C. 1962. La pensée sauvage. Paris, Plon.

Métraux A. 1958. Le Vaudou Haïtien. Paris, Gallimard.

Varagnac A. 1948. Civilisation et genres de vie. Paris, Albin Michel.

Vernant J-P. 2004. Les origines de la pensée grecque. Paris, PUF. 